$\mathrm{Oz}$

Volume 36

Article 7

$1-1-2014$

\title{
Embracing Complexity: Ecological Designs for Living Landscapes
}

Marta Brocki

PLANDFORM

Nina-Marie Lister

Ryerson University

Follow this and additional works at: https://newprairiepress.org/oz

Part of the Architecture Commons

(c) $($ ) $\Theta \Theta$

This work is licensed under a Creative Commons Attribution-Noncommercial-No Derivative Works 4.0 License.

\section{Recommended Citation}

Brocki, Marta and Lister, Nina-Marie (2014) "Embracing Complexity: Ecological Designs for Living Landscapes," Oz: Vol. 36. https://doi.org/10.4148/2378-5853.1530

This Article is brought to you for free and open access by New Prairie Press. It has been accepted for inclusion in Oz by an authorized administrator of New Prairie Press. For more information, please contact cads@k-state.edu. 


\title{
Embracing Complexity Ecological Design for Living Landscapes
}

\author{
Marta Brocki and Nina-Marie Lister
}

We are in the age of New Ecology. A paradigm shift in ecological thinking has unfolded over the last 25 years, and with it, a slow recognition of the inherent and fundamental complexity that shapes and defines our living world. Contemporary ecology now recognizes living systems as dynamic, complex, self-organizing, and, to some degree, unpredictable in their evolution and behavior. This characterization replaces earlier ecosystem models aligned with Newtonian determinism-mechanistic models that describe ecosystems through linear processes of succession through discrete states, increasing in structural diversity (biodiversity) and remaining in a steady ("climax") state until affected by disturbance from an external source. From this traditional perspective, ecosystem change is viewed as an aberration rather than an inevitable and inherently natural occurrence to which the ecosystem may in fact be habituated and adapted. This interpretation is not incorrect per se, but rather insufficient to cope with complexity. A linear interpretation of ecosystem evolution and behavior is limited in that it can not accommodate nor draw implications from the complexity inherent within living systems that occurs across multiple scales, from relationships between living organisms, among and between their environments, and in the adaptive processes that define resilient living systems.

A growing body of contemporary ecological literature-from concep-

tual to empirical-now describes living systems as complex and openended webs characterized by interrelationships that resist simplification through reduction to their component parts. ${ }^{1}$ When stability (or an apparent stable state) is observed within a living system, this observed stability is not uniformly and simultaneously present throughout the entire ecosystem, but rather in spatially and temporally discrete patches. ${ }^{2}$ The capacity for dynamic change and transformation is not only built into ecosystems, but in many cases, is integral to their growth, renewal, and long-term health.

Inherent to complexity theory, the concept of resilience refers to the degree to which a system is able to absorb and adjust to disturbances while still maintaining function and structural integrity. Resilience has both heuristic and empirical dimensions, arising from use in psychology, ecology, and engineering. As a heuristic, resilience refers to the ability of an ecosystem to withstand and absorb change to prevailing environmental conditions, and following these change events, return to a recognizable steady state (or a routinely cyclic set of states) in which the system retains most of its structures, functions, and feedbacks. Usually part of an ecosystem's normal dynamics, change events-or as C.S. Holling (1978) has called them, "surprises"are to some extent unpredictable, often causing sudden disruption to a system. Classic examples are forest fires (which are a necessary catalyst for the turnover and germination of seeds in certain environments), floods, storm events, or pest outbreaks, all of which can be exacerbated by external dynamics at larger scales. The ability of the system to withstand sudden changes assumes that behavior of the system remains within the stable domain (or regime) that contains this steady state in the first place. However, when an ecosystem shifts from one stability domain to another, losing many of its previously-existing structures and functions, it reorganizes via a bifurcation or "flip" in system states, called a regime shift. In cases of a regime shift, a more specific measure of ecosystem dynamics is needed for decision-making: that of ecological resilience. In this context, ecological resilience is a measure of the amount of change or disruption that is required to move a system from one state to another, and thus, to a different state being maintained by a different set of functions and structures than the former. A highly resilient ecosystem is therefore able to absorb a considerable degree of disturbance and adapt to the occurrence of a broad range of external pressures without being radically propelled into to a new configuration-one that may be unrecognizable or unenviable for its inhabitants and to which significant, potentially catastrophic, transformative adaptations must occur for survival. ${ }^{3}$

A rejection of the persistent and hierarchical dualism between culture and nature is necessary to fully incorporate these increasingly so- phisticated understandings within the creation and management of human-dominated environments. Contemporary ecology does not exclude humans from its understanding of living systems - rather, it encompasses the totality of interactions that take place among people and their physical, social, and experiential worlds. A new and fertile space to foster collaboration and synergy, in light of complexity, is revealed and functions under the premise that we are central participants in the design and management of our environments.

The structure of the contemporary urban (eco)system has been driven in tandem by the fields of engineering and urban planning-through physical intervention and regulatory frameworks respectively. ${ }^{4}$ In contrast to urban planning, engineering has largely operated without a substantial body of associated self-reflective critical theory. However, this has not rendered the efforts of the discipline removed from the reality of social and environmental consequences. Transportation networks, for example, have been deployed in a manner that maximizes efficiency and human mobility, but the consequences of which fragment the landscape, limiting mobility for non-human species seeking sustenance, shelter, and mates.

In this approach to city building, top-down control is implemented to produce an orderly, predictable, and stable environment-operating under the assumption that the city 
can be managed as a smoothly-operating machine. As the dominant lens through which the development of urban systems has been undertaken, this historic template continues to underlie and shape the relationship between landscape and built form within cities. $^{5}$

Grounded in a separation of land uses, this mechanistic approach to the management of urban lands has been enforced primarily using the Euclidian Zoning system. This approach and its associated epistemology, derived from Newtonian mechanics, based on order, control, and deterministic (predictable) outcomes, has been fundamental to the development and spatial distribution of built form and infrastructure in North America. Explicitly simplistic in its approach, arrangements of discrete, single, land-use categories (industrial, residential, commercial) form corridors through which transportation and utility networks are disseminated in order to facilitate movement, deliver services, and separate incompatible uses. The failure of this approach to account for the complex and evolving needs of urban spaces is documented by the abundance of project-specific planning tools designed to rectify its shortcomings. ${ }^{6}$

Engineered to service the urban grid, a network infrastructure of roads, bridges, tunnels, pipelines, sewers, and telecommunications underlies the functioning of urban spaces and serves two principal functions: to facilitate the horizontal movement of information, resources, and people; and to manage, structure, and contain elements of the environment. Most of the last century's urban development has been defined by civilengineering approaches to building infrastructure, applying an expeditious approach that favors maximum efficiency for minimal investment.

The resulting composition of urban form is characterized by infrastructural networks that are buried out of sight or externalized completely and spatially, and therefore, rendered invisible and discounted. An illusion of disconnect conceals the relationship between infrastructure, the users of its services, and the associated biophysical systems while simultaneously projecting the image of a landscape that remains undisturbed, in spite of substantial manipulation.? Parallel to this manufactured invisibility is a perceived containment of natural systems within infrastructural constraints. A fortification approach to development is intended to hold systems in a stable state and to allow urban environments to exist within a simplified reality removed from, and impenetrable to, the forces that we know act on natural systems.

Infrastructural networks often remain hidden until a failure in the system occurs and renders their inherent fragility visible (see Figure 1). ${ }^{8}$ Charles Perrow (1984) has observed that such failures are inevitable within complex systems and are more accurately called "normal accidents."
There is a growing recognition that traditional command-and-control interventions are often ineffective and that persistent over-management can have severe and unexpected consequences in the context of both natural and highly-urbanized landscapes. A catastrophic example can be found in the devastation caused by Hurricane Katrina in New Orleans. Over the course of many decades, New Orleans relied on flood-control based on fortification of drainage systems, and mitigation and externalization of storm water. Over time, intensive urbanization of floodplains, draining and conversion of wetlands, and an extensive network of dams and levees cumulatively resulted
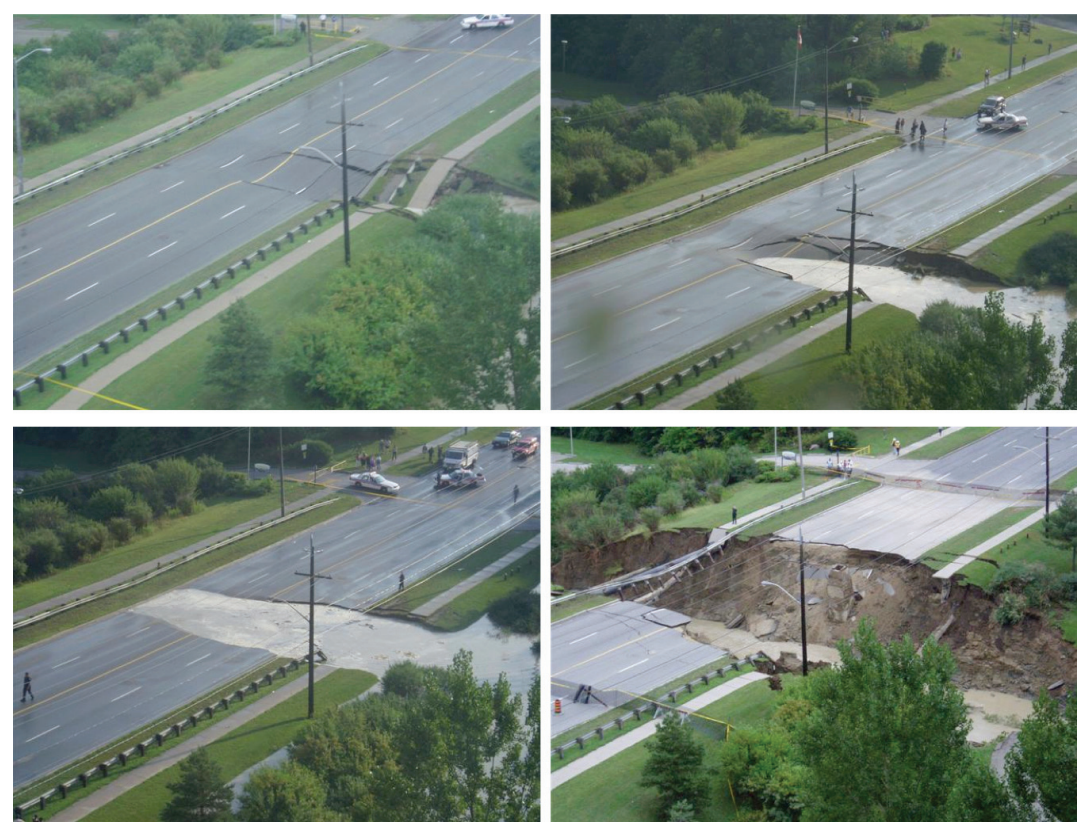

Figure 1. Road infrastructure wash-out caused by heavy rainfall. Photo: Lister. in an overloading of the ecological infrastructure that is integral to maintaining resilience through the absorption and diversion of large volumes of precipitation. Collectively, these interventions set the stage for a large-scale failure of the system to absorb an unprecedented amount of disturbance in the form of a Category 5 hurricane. ${ }^{10}$

Advancements in scientific knowledge in the field of ecology command that we adjust the way we approach interventions within the systems that sustain us to more accurately reflect how we have observed them to behave. This necessitates a transition away from attempts to enforce stabil- 


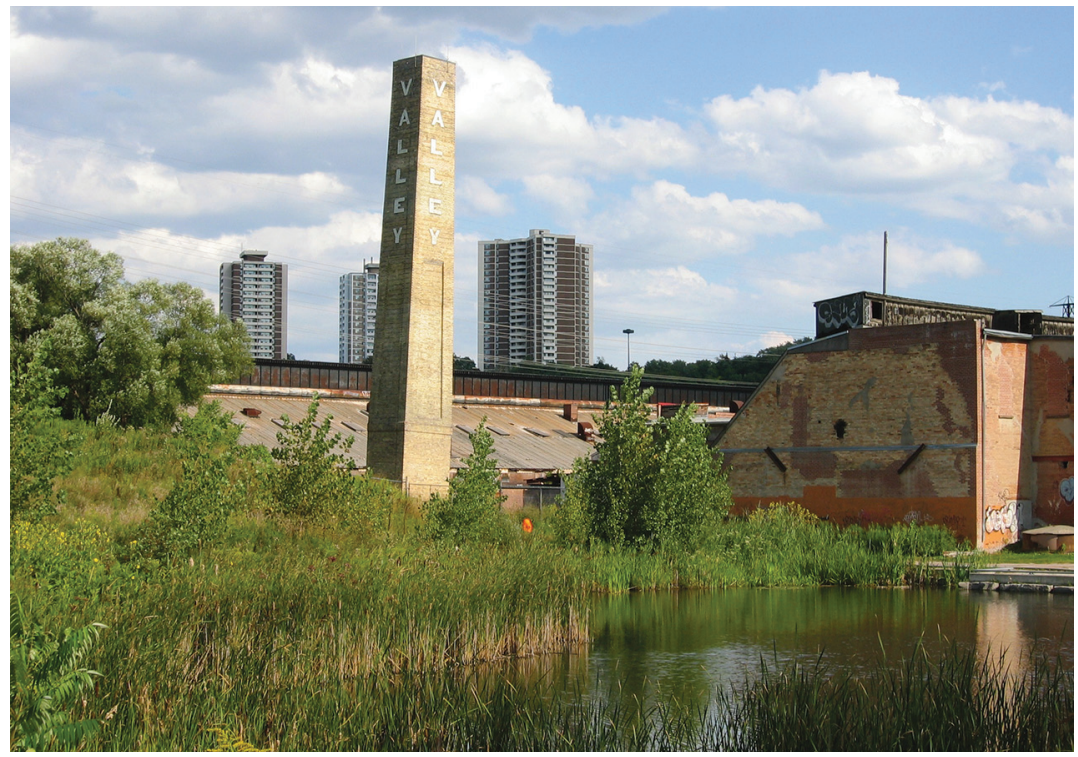

Figure 2. Constructed wetland at Evergreen Brick Works. Photo: Lister.

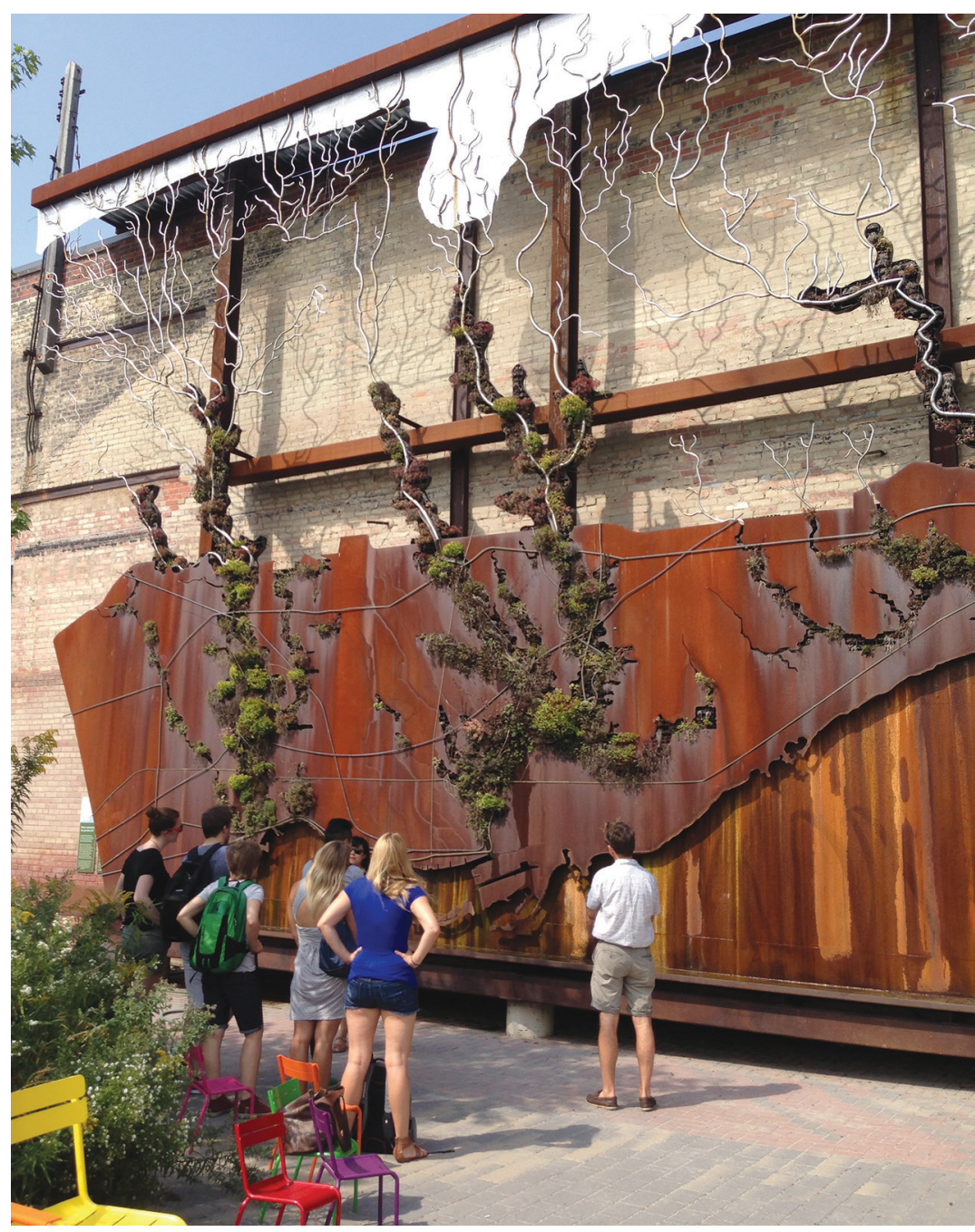

Figure 3. Ferruccio Sardella's "Water Consciousness" renders green infrastructure legiblethrough artistic representation of its function and invites visitors to consider their position within the watershed. Photo: Lister. ity and constancy to embracing complexity and fostering adaptation for improved resilience. Simultaneously a challenge and freedom afforded by the assimilation of complex systems thinking is that we cannot manage (eco)systems in their entirety. Our perceptions of stability can provide value only to the degree that they inform us about our cultural relationship to, and position within, the landscapes that sustain us. Using strategic design, urban systems can be guided in ways that allow for the enhanced absorption and facilitation of physical and cultural change while maintaining the functions we depend on. In other words, we can manage our own behaviors and interventions in these systems, but not the system itself as an object. In this sense, the concept of "environmental management" is at best, a problematic misnomer-and at worst, flawed hubris.

A movement towards an ecological urbanism, founded on synergistic relationships between natural and constructed systems, is imperative to create and maintain these environments into the future. In this context, the field of landscape infrastructure, with its essential ecological underpinnings, has emerged as a potent framework for the interpretation and reconceptualization of contemporary city building. Inherently crossdisciplinary, it draws on the domains of social, technological, visual, and ecological knowledge. Creating explicit connections between urban and natural realms positioned as confluent rather than dichotomous, it fosters an understanding of urbanism that acknowledges uncertainty and transition as manageable - and often necessary-rather than threatening.

The growing acceptance of flooding as a cyclical and inevitable occurrence in certain spaces is one area in which significant progress has been made in re-conceptualizing interventions from control to management.

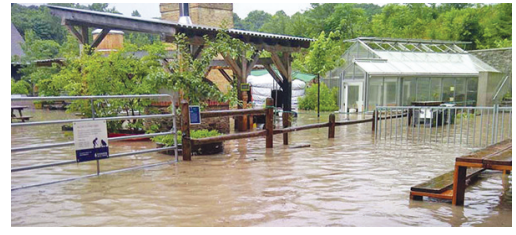

Figure 4. Evergreen Brick Works flooded. Photo: courtesy of S. Irvine.

This shift is beginning to be embodied in the planning frameworks of numerous coastal cities in North America (Toronto, New Orleans, New York) and on a smaller scale can be observed in site-specific adaptations.

The flood friendly landscape of Evergreen Brick Works in Toronto's Don Valley is conscious of its position within a floodplain along a major tributary of Lake Ontario (Figures $2-5)$. As a center for environmental education it fosters a hybrid ecology between its cultural function and its environment by engaging in a dialogue about what it means to exist in relation to the surrounding landscape in the heart of a metropolis, an extensive urban ravine system and watershed, a remediated brownfield, and geologically significant heritage site. Inherent in its design is an anticipation of storm events of varying intensities that, though temporarily disruptive, are not rejected but explicitly accommodated by the layout, form, and function of its structures. A constructed wetland created in place of the decommissioned quarry acts to filter water and mediate its quality as it passes through the site towards Lake Ontario, and contributes a beneficial ecological service functioning in support of the landscape rather than passively existing within it.

Projects such as the ARC International Wildlife Crossing Infrastructure Design Competition and the continued work of ARC Solutions ${ }^{11}$ to cultivate new approaches for understanding and building transportation 


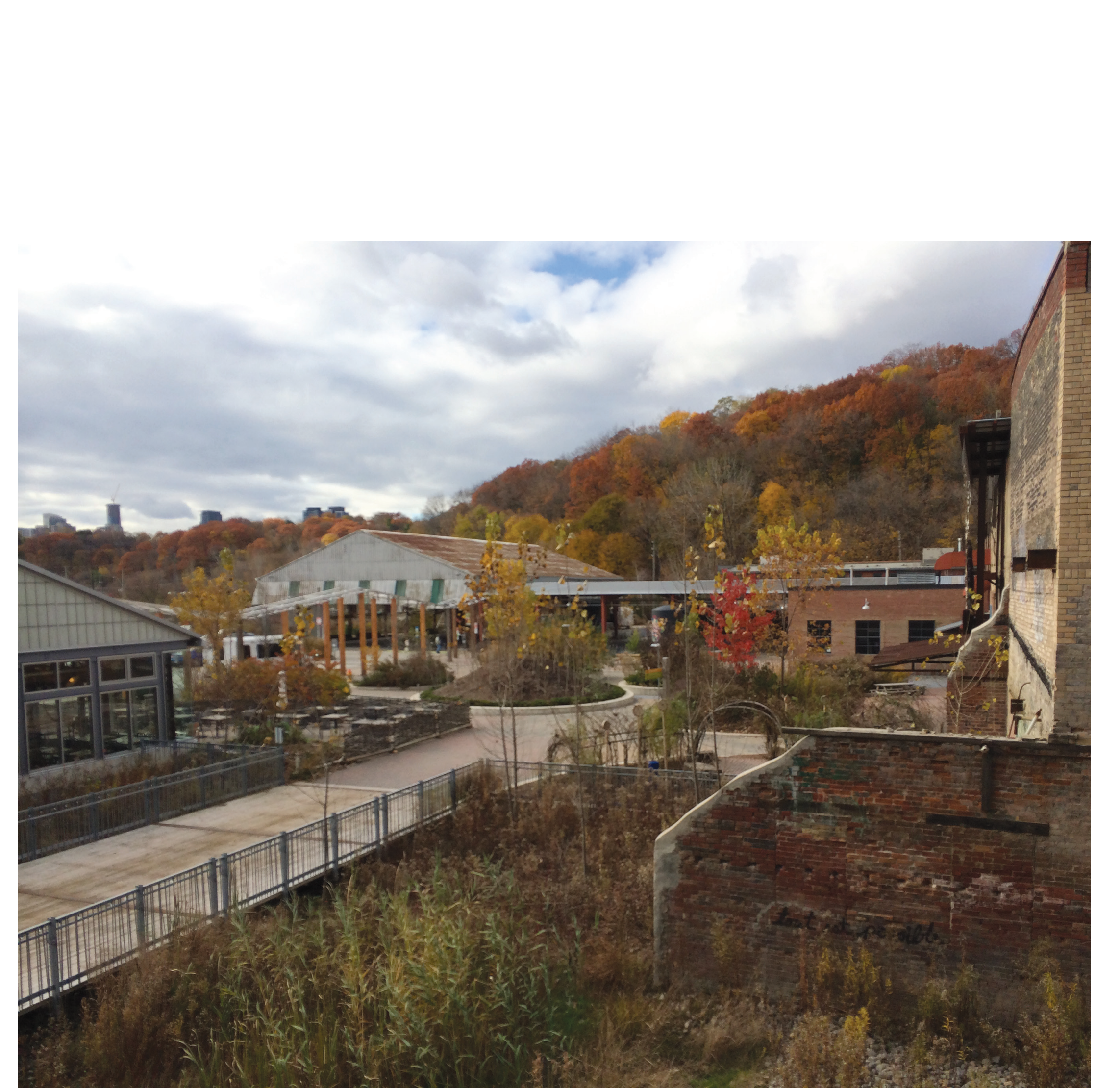



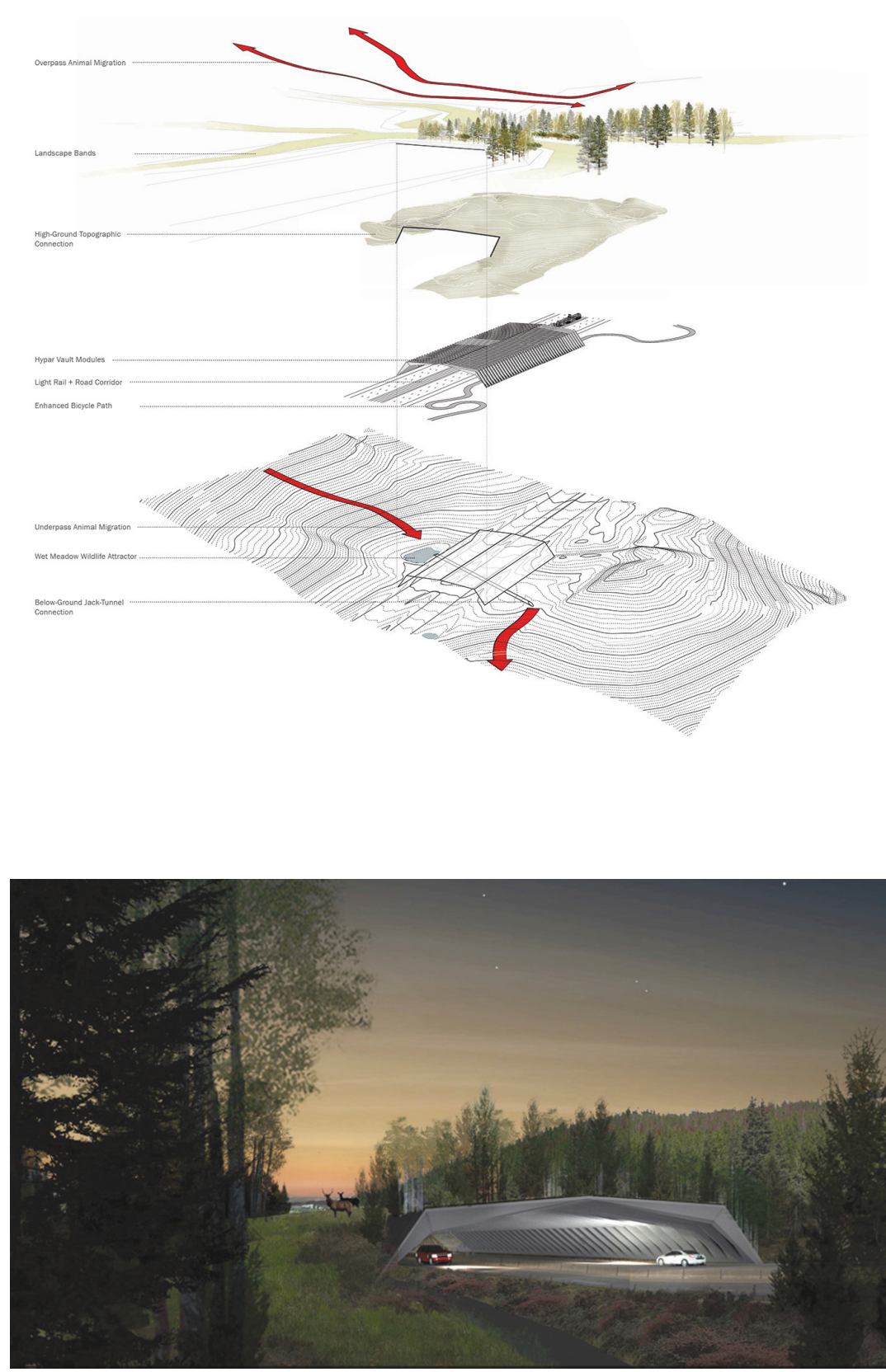

Figure 6. Stacking two worlds: The winning entry to the ARC International Wildife Crossing Infrastructure Design Competition reconnects the landscape above and below the road using modular precast elements and multiple designed habitat corridors. Photos: HNTB \& MVVA infrastructure focus on fostering synergy between human-centered uses and the landscapes in which they are embedded (Figure 6). By translating the perspective by which we intervene from a focus on triumphing over natural obstacles to one in which infrastructure functions with and for the surrounding landscape, a more resilient, multi-functional, and adaptive infrastructure is created.

The frequency and magnitude of major storm events have increased, and further shifts in climate and weather patterns are expected to become increasingly severe, affecting the distribution of arable lands and suitable habitats for humans and non-humans respectively. In this case, a rigid and unyielding transportation network may ultimately prove neither efficient or viable long-term. A complex systems-based design approach, practiced in interdisciplinary collaboration is urgently needed to achieve innovation in the delivery of radically new infrastructural networks-at all scales-from planning to spatial distribution, and from choice of materials to construction processes.

Designed ecologies have the potential to explicitly engage human and non-human dynamics in open-ended interventions that emphasize hybridity between environmental, social, and built outcomes. Dynamism is introduced through responsiveness to changing conditions intentionally incorporated into systems or through the potential for adaptation by way of movement and reconfiguration of structures over time. A clear avenue for the embrace of complexity and uncertainty is through the mediation of existing infrastructures: pathways that are vulnerable to pervasive, if not catastrophic, disturbance. Transportation, storm- and wastewater networks, power grids, and food systems are all ripe to benefit from rethinking, recasting and renewing the design approach from control-oriented to resilience-rooted. In the age of the New Ecology, on the doorstep of climate change and mass urbanization, we have an emerging awareness of the power of complexity and the insights that systems thinking and collaborative design may offer. Landscape infrastructure is uniquely positioned to overcome the limitations of externalization and invisibility in favour of embracing complexity and designing for resilience. 


\section{Notes}

1. The Canadian ecologist, C.S. Holling has been a pioneer in ecosystem complexity, with some of the earliest published research in applied ecosystem ecology. See e.g., Holling, C. S., "Resilience and Stability of Ecological Systems." Annual Review of Ecology and Systematics 4, 1973: 1-23. As early as 1971, Holling recognized the implications of complexity for resource management, and by extension, to planning and policy-making. See e.g., Holling, C. S. ed. Adaptive Environmental Assessment and Management. (London: John Wiley and Sons, 1978). His later work, together with colleagues at the University of Minnesota and Stockholm University, resulted in establishment of the Resilience Alliance (www.resiliencealliance. org) and the online journal Ecology \& Society. 2. See Chris Reed and Nina-Marie Lister, "Parallel Genealogies" in C. Reed and N-M. Lister, Eds., Projective Ecologies, pp. 18-34. (Boston, Barcelona: Harvard Graduate School of Design and ACTAR, 2014), and Nina-Marie Lister, "A Systems Approach to Biodiversity Conservation Planning," Environmental Monitoring and Assessment 49 (2/3), 1998: 123-155. for an extended discussion of stability and per- turbation as phenomenona inherent within living systems.

3. A discussion of applied resilience theory is found in Nina-Marie Lister, "Sustainable Large Parks: Ecological design or designer ecology?" In: J. Czerniak \& G. Hargreaves (Eds.), Large Parks. (Princeton NJ: Princeton Architectural Press, 2007), pp. 31-51. Design implications of resilience and associated drawings are found in Chris Reed and Nina-Marie Lister (Eds.), Projective Ecologies. (Boston, Barcelona: Harvard Graduate School of Design and ACTAR, 2014) 4. The work of Pierre Bélanger is instrumental to understanding the forces and processes that have led to the current distribution, arrangement, and form of land use and infrastructure within contemporary urban spaces. See: Bélanger, P. (2009). "Landscape as Infrastructure." Landscape Journal 28(1), pp. 79-95.; Bélanger, P. "Redefining Infrastructure." In M. Mostafavi, \& G. Doherty(Eds.), Ecological Urbanism (Baden, Switzerland: Lars Muller Publishers, 2010) pp. 332-349; Bélanger, P. (2012). "Landscape Infrastructure: Urbanism Beyond Engineering” In S. Pollalis, A. Georgoulias, S. Ramos, \& D. Schodek (Eds.), Infrastructure Sustainability and Design (pp. 276-315). New York: Routledge. 5. The metaphor of the machine and the influence of associated Newtonian determinism on Western culture and governance is wellestablished in a variety contemporary literatures ranging from environmental studies and political ecology to environmental history and organizational theory. See e.g., (respectively) Ann Dale, At the Edge: Sustainable Development in the 21st Century (Vancouver: UBC Press, 2001); Carolyn Merchant, The Death of Nature: Women, Ecology and the Scientific Revolution (New York: Harper Collins, 1980); and Margaret Wheatley, Leadership and the New Science: Learning About Organization from an Orderly Universe (San Francisco: BerrettKoehler, 1992).

6. Wickersham, J. (2001). "Jane Jacob’s critique of zoning. From Euclid to Portland and beyond." Boston College Environmental Affairs Law Review, 28 (4), 547-563.

7. Shannon and Smets describe this approach to building infrastructure as the "artifice of hiding” in Shannon, K., \& Smets, M., The Landscape of Contemporary Infrastructure (Rotterdam: NAi Publishers, 2013), p. 56.
8. Bélanger. P. (2010)

, Charles. Normal Accidents: Living With High-Risk Technologies. (New York: Basic Books, 1984)

10. In tracing the trajectory and revealing the limitations of management approaches affecting the Mississippi River delta, the work of Jane Wolff calls for a revised design paradigm that fosters legibility and fluidity through its activities: see www.guttertogulf.com and "Cultural Landscapes and Dynamic Ecologies: Lessons from New Orleans" In C. Reed and N-M Lister (Eds.), Projective Ecologies, (Boston, Barcelona: Harvard Graduate School of Design and ACTAR, 2014).

11. For more information visit www.arcsolutions.org

\section{Image Credits}

\section{Figures 1., 3.-5.}

Courtesy of author

Figure 2. Courtesy of HNTB + MVVA, 2010 Figure 6 . Courtesy of Irvine 